\title{
PROTOOPALINA DUBOSCQUI N. SP., OPALINE PARASITE D'UN POISSON MARIN
}

\author{
Par G. LAVIER
}

Les Opalines sont étrcitement adaptées dans leur parasitisme aux Amphibiens anoures ; aussi toute rencontre d'un de ces protistes dans un groupe zoologique différent revêt un intérêt particulier. Mais en fait jusqu'à présent, ces rencontres sont restées rares. Il y a déjà longtemps que Léger et Duboscq (1904) ont décrit une opaline du poisson marin Box boops L. ; c'était la première trouvée en dehors des batraciens, Opalina saturnalis qui fut rangée par la suite dans le genre Protoopalina Metcalf 1918. Puis fúrent signalées : Zelleriella piscicola da Cunha et Penido 1926, du poisson d'eau douce sud-américain Pimelodus clarias L. (Siluridé); Protoopalina nyanza Lavier 1927, d'un reptile africain Varanus niloticus L. ; Zelleriella boipeva Carini 1933, du serpent Ophis meremmii Wagler ; Zelleriella jægeri Carini 1933, du serpent Liophis jægeri (Günter) ; tout dernièrement enfin Wenrich (1935) relate la rencontre d'une Zelleriella chez un boa des Antilles.

Récemment, au cours d'un séjour au Laboratoire Arago, à Banyuls, j'ai eu l'occasion d'ouvrir deux capelans de Méditerranée, Gadus capelanus Risso; l'intestin des deux exemplaires présentait des Flagellés du genre Hexamita que j'ai décrits ailleurs ; un seul montrait en outre des Opalines appartenant au genre Protoopalina.

L'infestation par ces dernières était limité au rectum ; elle était peu intense et cela posait un problème : ne s'agissait-il pas d'une infection aberrante par Protoopalina saturnalis? le même coup de filet avait en effet ramené, avec les capelans, des bogues chez qui la présence de cette opaline est presque constante et il pouvait y avoir eu contamination entre les deux espèces vivant dans les mêmes eaux. Le fait n'est pas impossible : Wenrich, par exemple, note que l'opaline de son boa est morphologiquement identique à celle d'une rainette Hyla cinerea, proie habituelle de ce serpent et incline par suite à penser qu'il s'agit du même parasite ; Carini d'autre part remarque qu'Ophis meremmii vit dans les marais et se nourrit de grenouilles et de crapauds; on ne saurait guère penser toutefois que le capelan se fût infecté de la même manière, mais l'ingestion

Annales de Parasitologie, $\mathrm{T}$. XIV, $\mathrm{N}^{\circ} 3 .-1^{\mathrm{er}}$ mai 1936 , p. 272-277. 
de l'hôte n'est pas nécessaire et la simple cohabitation peut suffire grâce aux formes kystiques et même aux formes végétatives qui s'échappent du rectum des animaux parasités et peuvent être ingérées. Je puis en rapporter un exemple très typique : il y a plusieurs mois, recherchant des Cristispires dans l'intestin du mollusque Vivipara vivipara, j'ai eu la surprise, chez un individu, de rencontrer sur toute la longueur de l'intestin des opalines en petit nombre, il est vrai, mais parfaitement vivantes et mobiles ; dans les frottis colorés qui en furent faits, il était aisé de reconnaître des formes jeunes, présentant de deux à quatre noyaux, d'une Cepedea; elles provenaient sans le moindre doute de nombreux tềtards de diverses espèces qui étaient dans le même cristallisoir que les mollusques en attente.

Restait donc à comparer en détail le parasite du capelan et celui du bogue, seul animal marin actuellement connu pour héberger des opalines. Comme nous allons le voir, cette étude morphologique m'a amené à conclure qu'il s'agit de deux espèces voisines, mais non identiques.

Protoopalina saturnalis présente une assez grande variabilité que l'on peut schématiser, avec Léger et Duboscq, en formes allongées et formes ovoïdes, les premières mesurant de 150 à $250 \mu$ de longueur sur 20 à 30 de largeur, les secondes, en moyenne $100 \mu$ sur 60 . L'espèce du capelan, qui est plus petite, présente aussi un certain degré de variabilité, mais qui est loin d'être aussi accusé : j’ai noté pour la longueur un maximum de $142 \mu$, un minimum de $100 \mu$; la largeur varie de 40 à $43 \mu$; l'indice morphologique (quotient du chiffre de la largeur par celui de la longueur), calculé pour chaque individu, varie de 0,35 à 0,16 (par comparaison, celui de $P$. saturnalis varie, au minimum, de 0,6 à 0,14 ) ; en fait, on trouve surtout des individus moyens (fig. 2, 1 et 2) et, beaucoup plus rares, des individus allongés (fig. 2, 3) ou trapus (fig. 2, 4), comme dans beaucoup d'espèces d'Opalines. Le corps se termine toujours en arrière par un éperon net de 4 à $5 \mu$ de longueur, bien plus court par conséquent que celui de $P$. saturnalis (9 à $19 \mu$, Metcalf).

Les cils périphériques ont une longueur qui diminue progressivement, suivant leur point d'insertion, d'avant en arrière ; à l'extrémité antérieure, ils mesurent en moyenne de 7 à $8 \mu$, au maximum 10 , et sont donc beaucoup plus courts que chez $P$. saturnalis $(20$ à $25 \mu$, Léger et Duboseq) ; ils sont insérés sur des rangées spirales qui, dans un exemplaire moyen (fig. 1), sont au nombre de 16 et séparés par des intervalles de $3 \mu, 5$ dans la partie antérieure et de $4 \mu$ dans la partie moyenne et la partie postérieure. $P$. saturnalis a, d'après Léger et Duboseq, chez un individu moyen, 20 lignes spi-

Annales de Parasitologie, T. XIV, ${ }^{\circ} 3 .-1^{\text {er }}$ mai 1936.

18. 


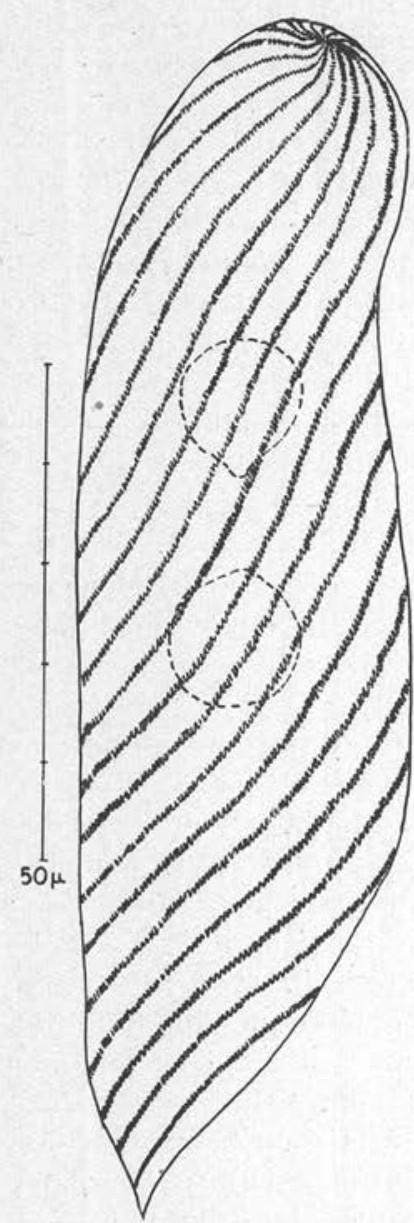

Fig. 1. - Protoopalina dubosqui n. sp. : lignes d'insertion ciliaires d'un individu moyen (exemplaire 2 de la figure 2 ). rales séparées par un intervalle de $3 \mu$, constant d'arrière en avant, variant toutefois pour Metcalf de 2,2 à $5,2 \mu$. $\mathrm{La}$ ciliature est toujours également fournie chez tous les exemplaires jusqu'à l'éperon terminal, et on n'observe pas chez l'opaline du capelan les individus à ciliature postérieure rare ou absente, qui sont si fréquents chez $P$. saturnalis.

L'ectoplasme présente une rangée d'alvéoles tassées les unes contre les autres, dont le fixateur contracte le contenu en sphérules; ces « ectosarcsphérules ", pour employer la nomenclature de Metcalf, sont constantes chez les protoopalines, mais elles sont ici particulièrement développées ( 2 à $5 \mu$ de diamètre), moins toutefois que chez $P$. saturnalis, où elles deviennent énormes ; et, par suite, l'épaisseur de l'ectoplasme est moins grande que chez cette dernière.

L'endoplasme contient des «sphérules » ovoïdes, de 2,5 sur $1 \mu$ en moyenne. Les deux noyaux sont sphéroïdes, mesurant de 12 à $15 \mu$ de diamètre, suivant leur état; il est très difficile d'ailleurs d'en trouver au repos, tant est grande l'activité mitotique ; après la télophase, la chromatine semble se disperser dans le noyau, mais on peut observer aussi une fu-

\section{Légende de la figure 2}

FIG. 2, - Protoopalina duboscqui n. sp. : 1-4, quatre exemplaires (fix. Bouin, col. hématoxyline ferr.); le pointillé représente l'extrémité des cils ; 1 et 2 , individus du type courant ; 3 , individu allongé ; 4, individu trapu ; 5-9, noyaux de cinq individus; la rangée supérieure correspond aux noyaux antérieurs ; 5, noyaux au repos encore unis par un filament (individu 4); 6, noyaux au repos (individu 2), dont l'un a une partie des chromosomes fusionnés en bande équatoriale incomplète ; 7 , noyaux dont le postérieur montre une plaque équatoriale typique ; 8 , anaphase ; 9 , deux noyaux à cinq chromosomes au lieu de dix ; 10, extrémité postérieure à gauche d'un individu allongé, à droite, d'un individu trapu. 

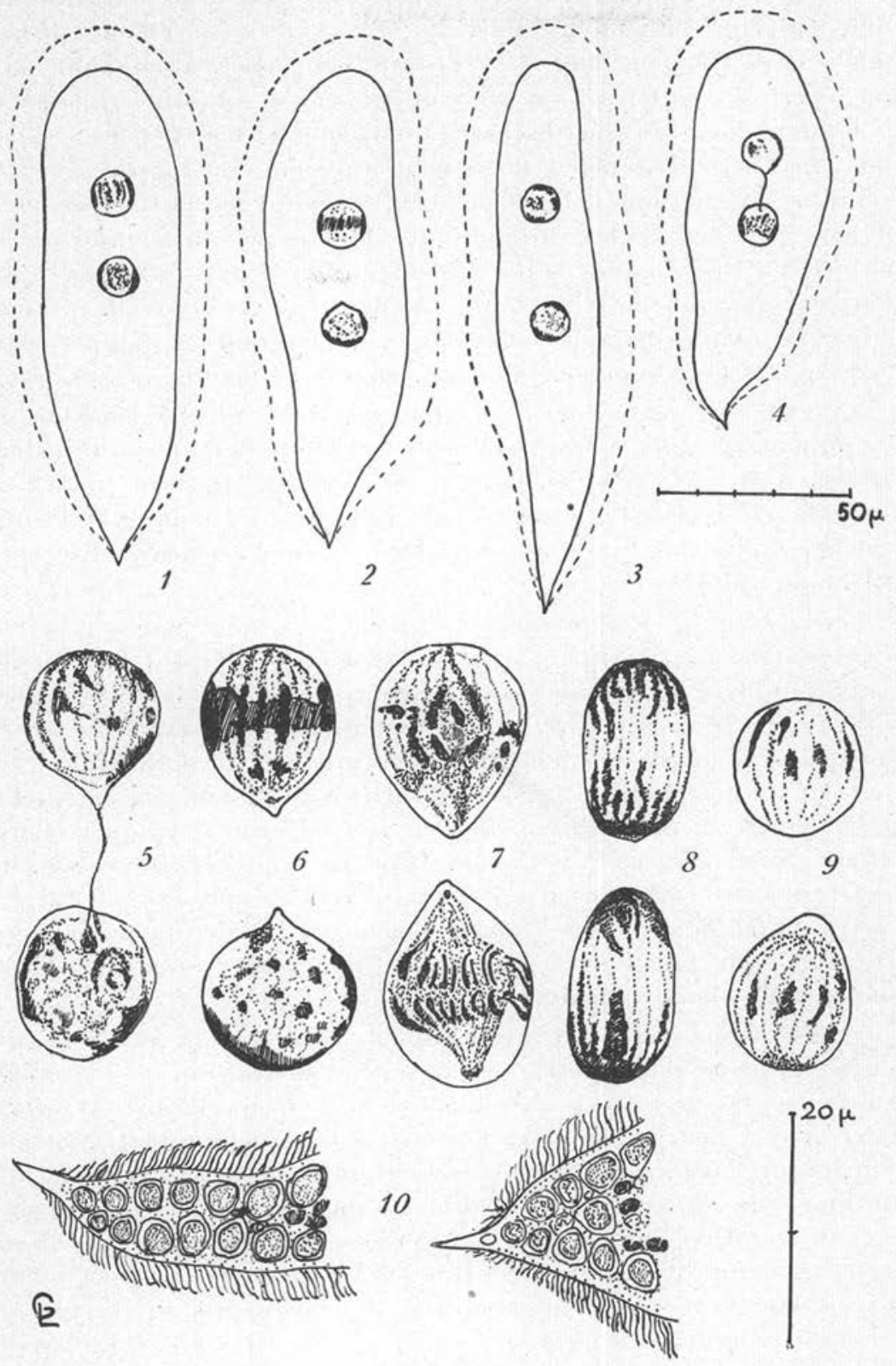

FIG. 2 
sion en bande équatoriale de plusieurs chromosomes (comparer les deux noyaux d'un même individu : fig. 2,6 ) ; c'est là un phénomène assez fréquent chez les Protoopalina et particulièrement marqué chez celle du bogue, qui présente ainsi un « anneau saturnien ». A la métaphase, j'ai pu observer, fait particulièrement notable pour une Opaline, l'existence d'une véritable plaque équatoriale avec clivage des chromosomes (fig. 2,7 ) et un fuseau d'aspect finement granuleux avec deux pôles chromatiques dont je ne saurais affirmer la nature centrosomienne ; le nombre des chromosomes est de dix, mais j'ai rencontré un individu (fig. 2, 9) dont les deux noyaux présentaient le nombre haploïde ; Metcalf a déjà signalé et figuré (1924, fig. 9, p. 21) un phénomène analogue chez Protoopalina intestinalis.

La structure, dans son ensemble, est donc proche de celle de $P$. saturnalis, mais, nous l'avons vu, un certain nombre de divergences sépare l'opaline du capelan de celle du bogue et justifie la création pour elle d'unẻ espèce nouvelle que je nommerai Protoopalina duboscqui, en hommage au professeur Duboscq, directeur du Laboratoire Arago.

Le capelan de Méditerranée est donc le second poisson marin à présenter un parasitisme par une Opaline ; en est-il l'hôte normal ou un hôte occasionnel ? et dans ce cas quel est l'hôte véritable? C'est ce que je ne puis dire ; je me contenterai de rappeler les réserves que m'inspirait au début la faible intensité de l'infection ; en tout cas, la morphologie permet d'écarter une infection accidentelle par l'opaline du bogue, seule espèce marine connue jusqu'à maintenant. Si d'ailleurs $P$. saturnalis avait quelque facilité à s'acclimater dans une autre espèce, il semblerait logique que ce fût en tout premier lieu chez Box salpa, voisin phylogénétiquement, herbivore comme lui, dont le milieu intestinal est très voisin, plutôt que chez un Gadidé carnivore.

Quoi qu'il en soit, il est intéressant de noter que le cas du bogue n'est plus isolé et peut-être par la suite d'autres espèces permettront-elles des découvertes semblables. Si l'on ajoute que $P$. saturnalis a une distribution bien plus large que ne le croit Metcalf (puisque je l'ai rencontrée à Arcachon chez tous les $B$. boops d'Atlantique que j'ai examinés et qu'il en est probablement de même partout où vit ce poisson, c'est-à-dire tout l'Atlantique-est et l'Océan indien), on voit qu'une recherche de ces Opalines de poissons serait susceptible de fournir d'intéressants renseignements sur la phylogénie de ce groupe de protistes. 


\section{BIBLIOGRAPHIE}

Carini (A.). - Zelleriella boipeve n. sp., parasite de l'intestin d'un serpent. C.R. Soc. de Biol., CXII, 1933, p.

Sur une nouvelle Zellerielle (Z. jageri) de l'intestin d'un serpent. Ibid., CXII, 1933, p. 1001.

Cunha (A. M. da) et Penido (J. C. N.), - Nouveau protozoaire parasite les poissons, Zelleriella piscicola n. sp. C.R. Soc. de Biol., XCV, 1926, p. 1003 .

Lavier (G.). - Protoopalina nyanza n. sp., Opaline parasite d'un reptile. C.R. Soc. de Biol., XCVII, 1927, p. 1709.

LÉGEr (L.) et Dubosce (O.). - Notes sur les infusoires endoparasites. Arch. Zool. exp. et gén., II, 1904, p. 337.

Metcalf (M. M.). - The Opalinid Ciliate Infusorians. U.S. Nat. Mus. Bull., 120, Washington, 1923.

Trends in evolution : a discussion of data bearing upon « Orthogenesis ». Journ. Morph. and Physiol., XLV, 1928, p. 1.

Wenrich (D. H.). - Host-Parasite relations between parasitic protozoa and their hosts. Proc. Amer. phil. soc., LXXV, 1935, p. 605.

Laboraloire de Zoologie el Parasilologie de la Faculté de médecine de Lille. 